PNL-SA-23193

\title{
A TUBE-EXCITED X-RAY FLUORESCENCE SPECTROMETER FOR USE IN SMALL-DIAMETER BOREHOLES
}

\author{
J. H. Reeves \\ R. J. Arthur \\ R. L. Brodzinski \\ C. L. Shepard
}

April 1995

Presented at the

Methods \& Applications of Radioanalytical Chemistry -III

American Nuclear Society

April 10-16, 1995

Kailua, Kona, Hawaii

Prepared for

the U.S. Department of Energy

under Contract DE-AC06-76RLO 1830

Pacific Northwest Laboratory

Richland, Washington 99352

\section{DISCLAIMER}

This report was prepared as an account of work sponsored by an agency of the United States Government. Neither the United States Government nor any agency thereof, nor any of their employees, makes any warranty, express or implied, or assumes any legal liability or responsibility for the accuracy, completeness, or usefulness of any information, apparatus, product, or process disclosed, or represents that its use would not infringe privately owned rights. Reference herein to any specific commercial product, process, or service by trade name, trademark, manufacturer, or otherwise does not necessarily constitute or imply its endorsement, recommendation, or favoring by the United States Government or any agency thereof. The views and opinions of authors expressed herein do not necessarily state or reflect those of the United States Government or any agency thereof. 


\section{DISCLAIMER}

Portions of this document may be illegible in electronic image products. Images are produced from the best available original document. 


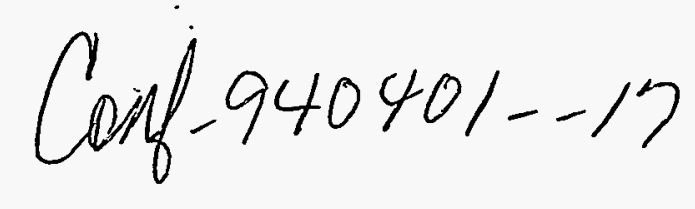

\title{
A TUBE-EXCITED X-RAY FLUORESCENCE SPECTROMETER FOR USE IN SMALL-DIAMETER BOREHOLES
}

\author{
J. H. REEVES, R. J. ARTHUR, R. L. BRODZINSKI, C. L. SHEPARD \\ Pacific Northwest Laboratory, Richland, Washington 99352 (USA)
}

\begin{abstract}
A portable in-situ $x$-ray fluorescence analytical system that uses an $x$-ray tube excitation source and a cooled $\mathrm{Si}(\mathrm{Li})$ spectrometer for detecting characteristic emission $\mathrm{x}$ rays has been developed for use in small-diameter wells and boreholes. The 15-watt, iron-anode x-ray tube operates up to $30 \mathrm{kV}$. Three wells at the Sandia National Laboratory Chemical Waste Landfill, lined with $76 \mu$ thick polyethylene, were logged specifically for $\mathrm{Cr}$ contamination. Detection limits below $50 \mathrm{ppm}$ were achieved with counting intervals of 600 seconds and with the $\mathrm{Si}(\mathrm{Li})$ detector operating at $450-\mathrm{eV}$ resolution (full width at half maximum [FWHM] for the Mn K-alpha $\mathrm{x}$ ray).
\end{abstract}

X-ray fluorescence (XRF) is an accepted method for in-situ analysis of soils and sediments for metal contaminants. Field portable systems are currently being used for surface (and near surface) analyses and deliver acceptable results for some metal contaminants at the level of concern ${ }^{(1-2)}$. The immediate determination of contaminant concentrations may allow concurrent remedial actions to be conducted. There are many sites where the contaminants are not located at or near the surface. A down-well XRF analytical system could be utilized at some of these locations to provide quantitative results faster and cheaper than any other analytical method, including field portable XRF systems. Since XRF analyzes a relatively small sample volume, considerable effort is required to insure that the sample is representative of the macro environment. For analysis by field portable or laboratory based systems, this is usually accomplished by homogenizing a large volume of the sediments and then analyzing several aliquots to insure homogeneity, accuracy, and representation. This procedure requires significant time and effort and makes the cost of each analysis relatively expensive even for the comparatively inexpensive procedure of XRF. A downwell in situ probe can make a statistically significant number of measurements of a few seconds each at any location in the borehole to quantify the contaminants of interest without the odious tasks of sample collection, homogenization, sample preparation, and waste disposal, and all at a cost of only dollars per measurement.

Accurate analysis of data generated with XRF spectrometers can be difficult if a large suite of elements are present in the material being analyzed. The detection limit is greatly improved with the use of high resolution detectors. The ultimate goal of our research is to develop an easily transportable, light-weight, down-well, spectrometer using a high resolution $\mathrm{Si}(\mathrm{Li})$ detector which will operate at count rates up to 50,000 counts per second (cps) at low dead time and deliver detection limits below $10 \mathrm{ppm}$ for all metals having atomic numbers above titanium with 100 -second counting intervals.

Chromium is one of the elements of interest for this project and is considered to be one of the more difficult to measure in soil containing almost $1 \%$ iron due to 
interference from the characteristic iron $x$ rays. The high density polyethylene liner required to keep the borehole from collapsing further degrades the spectra. Therefore, our research has concentrated on chromium, but many of the other elements can be measured with this technique once it has been sufficiently developed. Results from our early efforts toward these goals are reported, and the second phase, now under development, is discussed. Excitation radiation from both a radioactive source $\left({ }^{109} \mathrm{Cd}\right)$ and from an $\mathrm{x}$-ray tube were evaluated. A demonstration involving three wells (UCAP-1, UCAP-2, and UCAP-3) located near the chromic acid pit in the chemical waste landfill at Sandia National Laboratory was performed in September 1993.

\section{Experimental}

In the first phase of this effort a $200-\mathrm{mm}^{2} \mathrm{Si}(\mathrm{Li})$ detector contained in a $6.35 \mathrm{~cm}$ diameter by $91.4 \mathrm{~cm}$ long liquid nitrogen cooled cryostat having a holding time greater than 16 hours was used. The amplifier, high voltage power supply, analog-to-digital converter (ADC), multichannel analyzer (MCA), and front-end computer were located adjacent to the cryostat inside a $8.89 \mathrm{~cm}$ diameter housing into which the ${ }^{109} \mathrm{Cd}$ source could also be attached. Electrical power was supplied through umbilical cables that also contained the wires for passing spectra to an above-ground computer for data analysis and storage (Fig. 1). Overall length of the system was $\approx 2 \mathrm{~m}$.

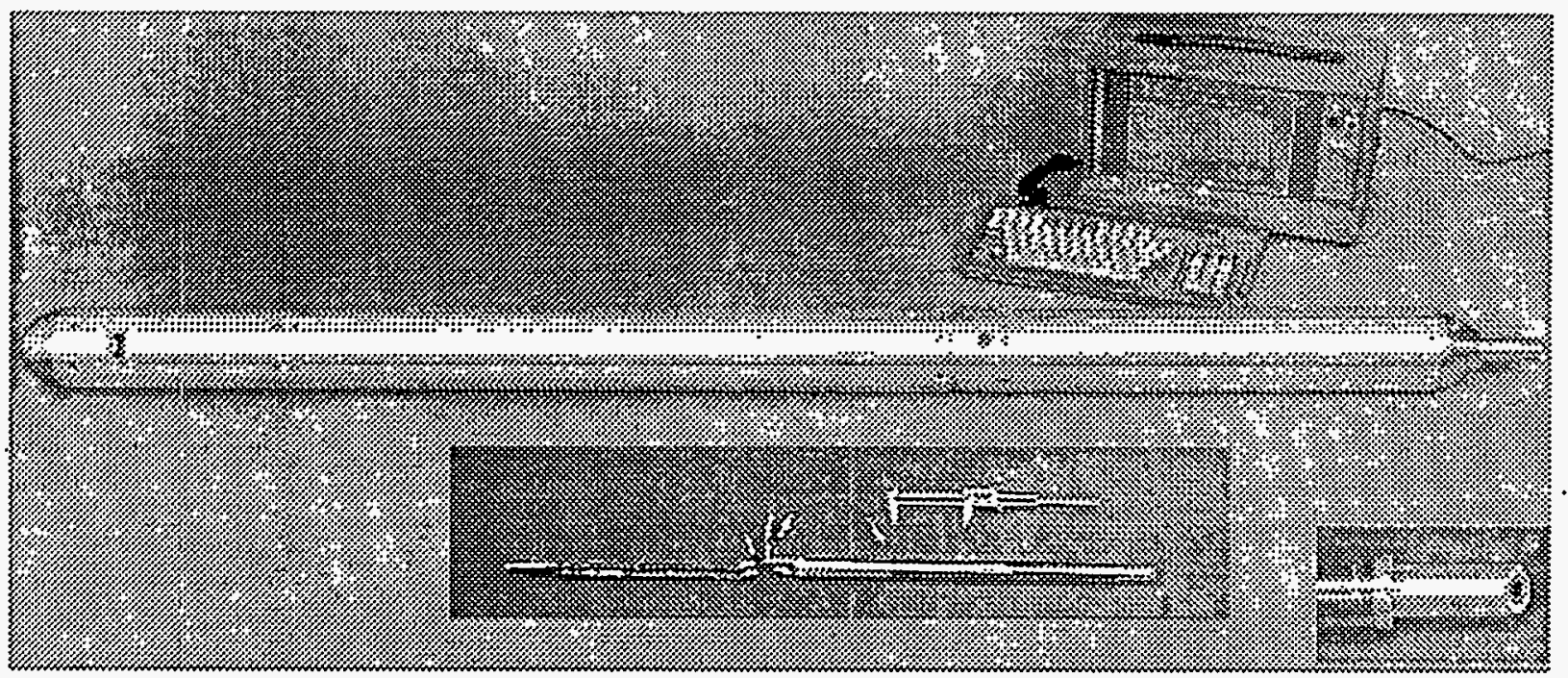

Fig. 1 Portable in situ x-ray fluorescence analysis systems for small diameter boreholes that use $\mathrm{x}$-ray tube excitation sources and liquid nitrogen cooled $\mathrm{Si}(\mathrm{Li})$ spectrometers for detecting characteristic emission $x$ rays. The insert in the lower right hand corner shows the beryllium window for the characteristic $x$-rays received by the smaller spectrometer 
The $\mathrm{Si}(\mathrm{Li})$ detector was adjacent to a $0.05-\mathrm{mm}$ thick beryllium window in the cryostat. A $2.54 \mathrm{~cm}$ square hole located in the end section of the housing was covered with a 0.38$\mathrm{mm}$ thick beryllium window and provided a path for the excitation photons and for the characteristic fluorescence $\mathrm{x}$ rays produced in the soil. The well was lined with $76 \mu$ thick polyethylene.

The end section was replaced with a section containing the $\mathrm{x}$-ray tube whenever the tube was used as the excitation source. The tube was positioned such that excitation $\mathrm{x}$ rays penetrated the $0.38-\mathrm{mm}$ thick beryllium window and struck the soil at a similar angle to that of the ${ }^{109} \mathrm{Cd}$ source $\mathrm{x}$ rays. The 15 -watt (max) iron-anode $\mathrm{x}$-ray tube was $\approx 3.2 \mathrm{~cm}$ in diameter by $\approx 17 \mathrm{~cm}$ long. Voltage up to $30 \mathrm{kV}$ can be applied to the tube. The maximum current is 1 milliampere for voltages up to $15 \mathrm{kV}$. For voltages higher than $15 \mathrm{kV}$, the current must be reduced to avoid exceeding the tube's 15-watt capacity.

Although the detector offers $220-\mathrm{eV}$ resolution when operated in the laboratory environment with normally available electronics, our down-well system only achieved a resolution of $450 \mathrm{eV}$. Our efforts to build electronics that would handle count rates greater than $20,000 \mathrm{cps}$ while maintaining high resolution were not successful before the scheduled demonstration reported on here.

Also shown in Fig. 1 is the second generation spectrometer containing all the above components in a 3.1-cm diameter housing, $\approx 1 \mathrm{~m}$ long. The electronic section is shown removed from the housing.

\section{Results}

Data were collected with the $x$-ray tube anode current set at $0.06 \mathrm{~mA}$ and high voltage at $19.1 \mathrm{kV}$. The isotopic excitation data were collected using a $14-\mathrm{mCi}{ }^{109} \mathrm{Cd}$ source. Thin aluminum was used to filter out low energy $x$ rays, and wide-angle collimators were used. Spectra discussed in the following sections will be referred to simply as "tube" or "source" for purposes of identifying the excitation radiation. A 5-minute "tube" spectrum corresponding to a chromium concentration of $670 \mathrm{ppm}$ was obtained at a depth of 17.4 $m$ in well UCAP-3 at a counting rate of about $10,000 \mathrm{cps}$ and is shown in Fig. 2. For comparison, a 10-minute "source" spectrum collected at the same depth and a counting rate of about 5,000 cps (our down-well electronics could not process the higher energy pulses as efficiently as it handled the lower energy pulses, which resulted in a lower count rate for a given dead time) is also shown.

As can be seen in Fig. 2, much of the activity in the "tube" spectrum is in the vicinity of the iron $\mathrm{K}$-alpha energy at $6.400 \mathrm{keV}$. X rays at this energy have a high probability of being absorbed by chromium which then emits characteristic $5.412 \mathrm{keV} \mathrm{K}$-alpha $x$ rays. Conversely, most of the activity in the "source" spectrum is located near the $22.101 \mathrm{keV}$ $\mathrm{K}$-alpha line of ${ }^{109} \mathrm{Ag}$. $\mathrm{X}$ rays at this energy have a low probability of being absorbed by chromium.

Figure 3 shows a comparison of a portion of the "tube" spectrum from Fig. 2 obtained with the $450 \mathrm{eV}$ resolution spectrometer and a state-of-the-art $135 \mathrm{eV}$ resolution spectrometer recently assembled. Obviously, the system with better resolution leads to improved detection limits. 


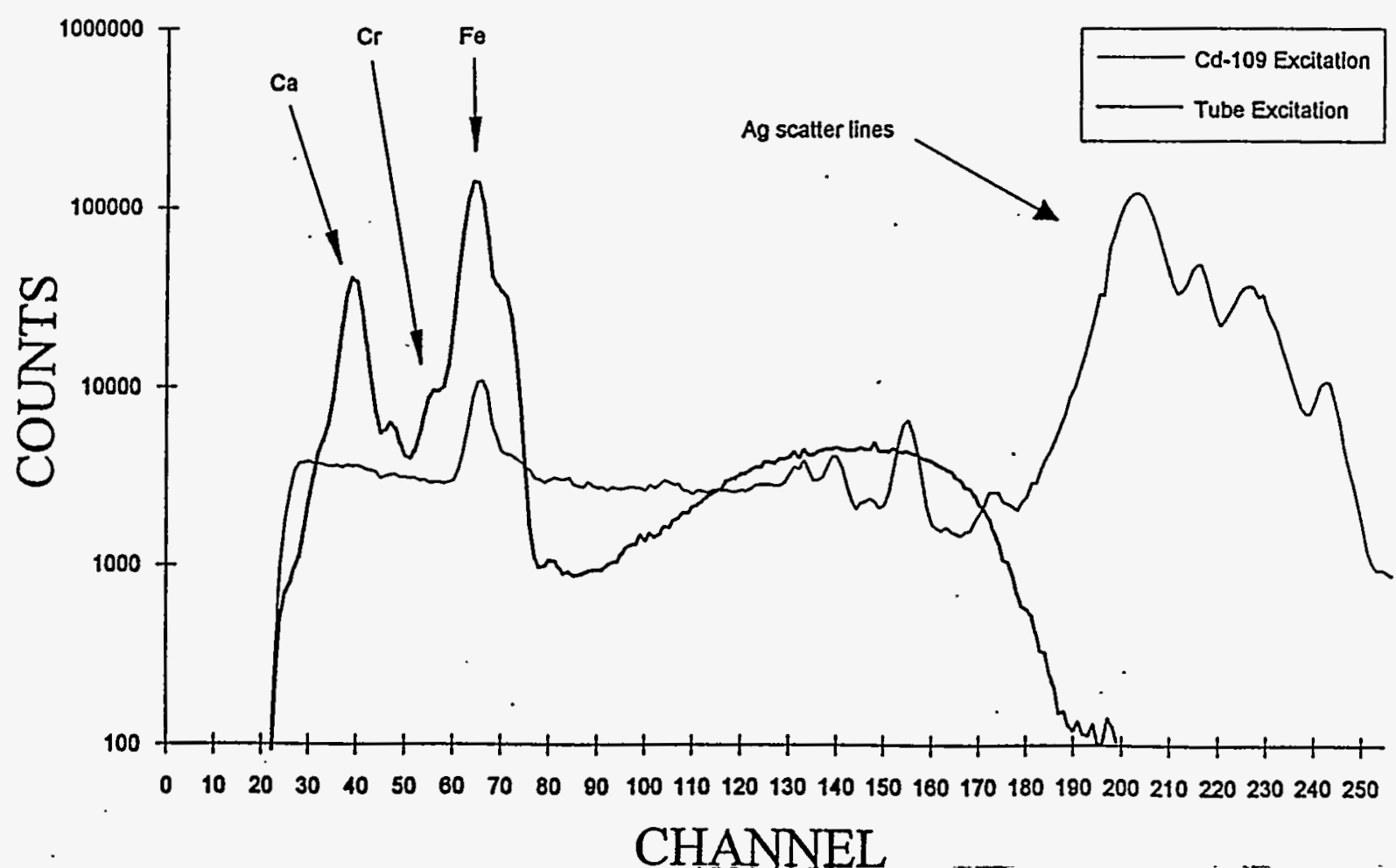

Fig. 2 Comparison of a "tube" spectrum obtained at a depth of $17.4 \mathrm{~m}$ in well UCAP-3 at a counting rate of $\approx 10,000 \mathrm{cps}$ and a "source" spectrum collected at the same depth and a counting rate of $\approx 5,000 \mathrm{cps}$

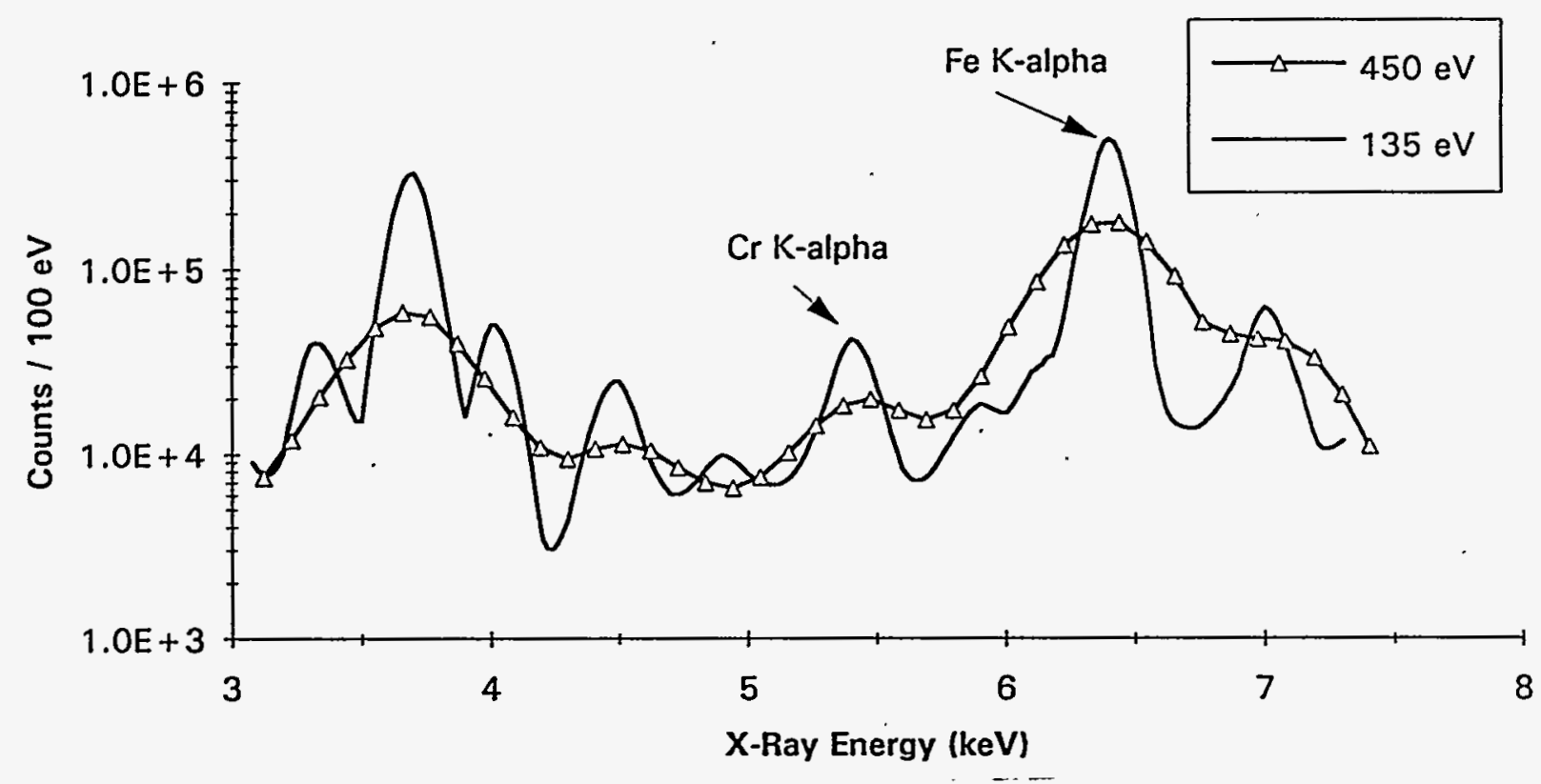

Fig. 3 Comparison of "tube" spectra using the original $450 \mathrm{eV}$ resolution spectrometer and a state-of-the-art $135 \mathrm{eV}$ resolution spectrometer 
Concentrations of chromium were determined by comparison of the net peak area. observed at the chromium K-alpha energy in well logged spectra to that observed in standards prepared by adding known amounts of chromium to soil obtained at the Sandia site. The standards were measured at the same voltage and current settings at which the well logging spectra were acquired. No corrections have been made for particle size differences or any other phenomena at this time.

Three 12-gram aliquots of soil taken at random from $300 \mathrm{~kg}$ of background soil collected at the Sandia site were measured for chromium by neutron activation analysis. The results $(27,30$, and $25 \mathrm{ppm})$ imply the chromium concentration is reasonably consistent. This does not guarantee that the background concentration of chromium is constant at various depths in any given well, but it is in general agreement with the lowest values obtained with our down-well XRF system.

Figure 4 shows the chromium concentrations from both the "tube" and "source" measurements as a function of depth in well UCAP-3. Each data point represents the results of a single measurement rather than the average of several measurements at each depth. The results are therefore subject to sampling errors in addition to the large statistical uncertainties encountered near the detection limit. Nevertheless, the values are in general agreement with each other and with the results based on Inductively Coupled Plasma analyses. The detection limit (68\% confidence limit) for chromium obtained with the "source" is $\approx 300 \mathrm{ppm}$ while that obtained with the "tube" is $\approx 50 \mathrm{ppm}$.

\section{Discussion}

Tubes in the kilowatt range are commonly used in laboratory based XRF systems, but these generally require water cooling, which is not convenient in down-well operations. Our measurements show that a low power tube can be used down-well with only ambient cooling and can provide adequate $\mathrm{x}$-ray intensity for primary excitation. Chromium concentrations were measured down to nearly background levels in all three test wells drilled in the Chemical Waste Landfill at Sandia National Laboratory.

Due to electronic difficulties during our demonstration, optimum operating parameters (for the measurement of chromium) of $\approx 12 \mathrm{kV}$ and $0.6 \mathrm{~mA}$ could not be achieved. Nevertheless, the "tube" data were much superior for the detection of chromium than the ${ }^{109} \mathrm{Cd}$ "source" data. An isotopic source of sufficient activity, appropriate energy, and appropriate geometry would, of course, yield results similar to the "tube" for the detection of chromium. However, having a radioactive source with the correct energy for optimum sensitivity for every element of interest in any particular matrix is not practical. Obtaining the necessary authorization for transportation, storage, and use of several radioactive sources is difficult and time consuming. Since the tube can be turned "off" when not in use down-well, it is an intrinsically safe device, and problems associated with transportation and handling of radioactive material are limited to issues associated with a radiation-generating device.

The very low power $(\approx 1$ watt) needed to saturate our counting system at $\approx 10,000 \mathrm{cps}$ indicates that if a multielement tube could be manufactured with remote anode, voltage, and current selection, and with appropriate remotely selectable filters and collimators, 


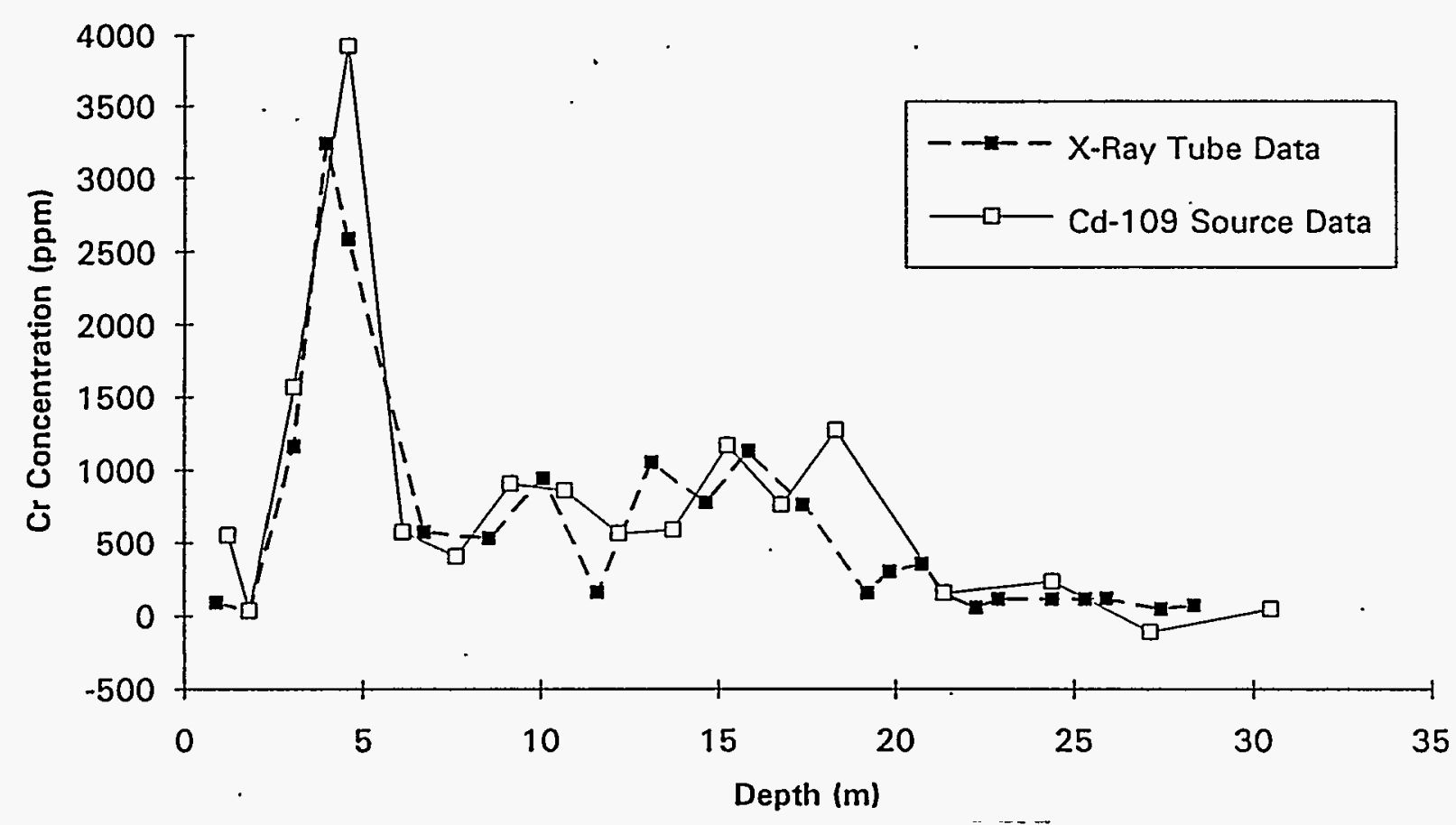

Fig. 4 Comparison of the in situ chromium concentrations measured with both "source" and "tube" excitation as a function of depth in well UCAP-3

near optimum parameters could be obtained for all elements of interest in any particular environment. The second phase of this development consists of a down-well, liquidnitrogen-cooled, $135 \mathrm{eV}$ resolution, $\mathrm{Si}(\mathrm{Li})$ detector capable of fitting inside a cone penetrometer $3.17 \mathrm{~cm}$ in diameter and a miniaturized version of the electronics necessary to process the pulses at rates up to $10,000 \mathrm{cps}$ while maintaining the design resolution of the detector. Laboratory tests with this detector and electronics are currently in progress. The third phase of the program will produce a "tunable" $x$-ray tube, as described above, in order to optimize the sensitivity and specificity for detecting any element or suite of elements in a given environmental scenario and an electrically cooled characteristic $x$-ray detector to eliminate the need for liquid nitrogen.

This work was supported by the U. S. Department of Energy (DOE) under Contract DE-AC06-76RLO 1830. Pacific Northwest Laboratory is operated for the DOE by Battelle Memorial Institute.

\section{References}

1. "Field Portable X-Ray Fluorescence," U. S. EPA Quality Assurance Technical Information Bulletin, Vol. 1, No. 4, May, 1991.

2. M. BERNICK, P. F. BERRY, G. R. VOOTS, G. PRINCE, J. B. ASHE, J. PATEL, P. GUPTA, Adv. X-Ray Anal., 35 (1992) 1047. 